\title{
Proactive condition-based bridge rehabilitation planning including LCA and LCC
}

\author{
Gordt A. ${ }^{1}$, Maier S. ${ }^{1}$, Henzler K. ${ }^{1}$, Camarinopoulos S. ${ }^{2}$, Kallidromitis \\ V. ${ }^{3}$, Sanna C. ${ }^{3}$, Panetsos P. ${ }^{4}$, Karali T. ${ }^{5}$ \& Bouklas K. ${ }^{6}$ \\ 1 University of Stuttgart, Germany \\ ${ }^{2}$ RISA Sicherheitsanalysen $\mathrm{GmbH}$, Germany \\ ${ }^{3}$ T.E.C.N.I.C. tecniche e consulenze nell'Ingegneria Civile Consulting Engineers, \\ Italy \\ ${ }^{4}$ EOAE Egnatia Odos, Greece \\ ${ }^{5}$ Environmental Reliability \& Risk, Greece \\ ${ }^{6}$ Institute of Communication \& Computer Systems (ICCS), Greece
}

\begin{abstract}
The implementation of structural health monitoring (SHM) for management and maintenance of critical transport infrastructures, such as bridges, dams or tunnels, is a widely established approach. Even though, SHM shows various technical limitations (e.g. relating to spatial capabilities of the sensors, high cost, repeatability or interpretation of the sensor measurements to support structural assessment and prediction of the infrastructure condition states). Furthermore, linking SHM with life cycle based methodologies such as life cycle costing (LCC) or life cycle assessment (LCA) is only recently discussed. The SENSKIN EC cofunded research project aims to overcome above mentioned challenges through development of a new sensor system and its integration within a Decision Support System (DSS) for proactive condition-based structural rehabilitation planning during the bridge life cycle. The DSS will include structural assessment models (exclusively based on sensor measurements for assessing the bridge condition and damage states of the main structural and a rehabilitation planning module (RPM) that will enable end-users to assess the life cycle economic and environmental implications of bridge rehabilitation options. Hereby, a tailored submodule for integrated life cycle costing (LCC) and life cycle assessment (LCA) assists, taking into account not only direct impacts of the rehabilitation solutions but also external effects caused by restricted traffic conditions (e.g. due to ongoing construction works). Thus, the SENSKIN project will contribute to a sustainable infrastructure. The following paper will sketch out the main scientific and functional structures of the developed DSS, with focus on the RPM and its LCA/LCC submodule for bridge rehabilitation planning.
\end{abstract}

Keywords: Life cycle assessment (LCA), life cycle costing (LCC), proactive decision support, condition-based bridge maintenance, rehabilitation planning 


\section{Introduction}

Transport infrastructures are a vital part of the whole logistics in Europe and their importance is steadily increasing - traffic is not only expected to rise due to the growing population and motorisation, but also due to the intensifying trade relations and associated road-based transportation of goods. The higher traffic demand will pressure on the current transport infrastructure which is in many cases already experiencing deterioration, e.g. bridges are ancient since they are built to stand for several decades. Moreover, climate changes and consequential extreme environmental conditions are predicted to negatively impact on these existing infrastructures, requiring continuous maintenance in order to retain their condition and safety.

The implementation of structural health monitoring (SHM) for management and maintenance of critical transport infrastructures, such as bridges, dams or tunnels, is hereby a widely established approach. However, due to their sensors, SHM technologies show various limitations which are related e.g. to spatial capabilities of the sensors, high cost, durability aspects, repeatability or the interpretation of the sensor measurements to support structural assessment and prediction of the infrastructure condition states. With regard to decision support from an environmental or cost perspective and for proactive condition-based bridge rehabilitation planning, linking SHM with life cycle based methodologies such as life cycle costing (LCC) or life cycle assessment (LCA) is only recently discussed [1], [2], [3].

\subsection{The SENSKIN project}

For the purpose of improving current practice in structural health monitoring (SHM) for maintenance and rehabilitation planning of transport infrastructures, the SENSKIN project aims to develop a new sensing device (skin like SENSKIN sensor) which is able to detect even small strains in the surface area of infrastructures. The sensing device will be coupled with a data acquisition system (DAQ) to digitize sensed strains, a communication module for implementing communications operational logic and data transmission, a processing module as computational core, a low power wakeup receiver as well as power management, energy harvesting and energy storage units, to form the resilient SENSKIN Integrated System. Power supply of this integrated system will be realized via a photovoltaic panel that provides the highest power concentration $\left(100 \mathrm{~mW} / \mathrm{cm}^{2}\right)$.

Furthermore, a dedicated decision support system (DSS) will be set up for bridge rehabilitation planning purpose. The DSS will be capable to handle the data generated by the SENSKIN sensor for condition-based structural assessment. From a life cycle perspective, the DSS will be enhanced by the integration of life cycle costing (LCC) and life cycle assessment (LCA) functionality. Thus, the DSS will support and facilitate the development of sustainable bridge rehabilitation planning and respective holistic intervention, based on precise actual structural condition state predictions of the assessed transport infrastructure.

The SENSKIN project is co-funded by the sustainable EU Horizon 2020 Research and Innovation programme and its developments are strongly driven by improving 
the assessment of transport infrastructures, life cycle optimisation considerations as well as industrial end-user application.

\section{Proactive decision support via LCA and LCC}

As a first step within the scope of the SENSKIN project, a methodology for decision-making on structural bridge rehabilitation is being developed. This methodology takes into account the precise actual condition of the bridge construction, life cycle cost analyses (LCC) and life cycle assessment (LCA) results of available rehabilitation options. Besides cost and environmental implications from bridge construction, the cost and environemntal consequences due to external effects will be evaluated. In a second step, the methodology will be implemented as part of a software system called the Rehabilitation Planning Module (RPM). The RPM is combined with an expert system for risk-based decision-making on the type (WHAT) and timing (WHEN) of intervention in bridge rehabilitation. The expert system links directly to the SENSKIN sensor technology and to information from the new SENSKIN bridge monitoring system (SENSKIN Integrated System) as well as to historical, external and knowledge databases. The RPM and the expert system constitute the Decision Support System (DSS), which offers bridge operators rehabilitation options for proactive interventions under operating loads and interventions after extreme events depending on the actual damage condition and taking into account life cycle considerations (costs, environment).

\subsection{The SENSKIN Rehabilitation Planning Module (RPM)}

The RPM consists of various modules which together contribute to the decisionmaking process in bridge rehabilitation. In total four different modules (Figure 1) contribute to the life cycle related assessment of bridge maintenace measures.

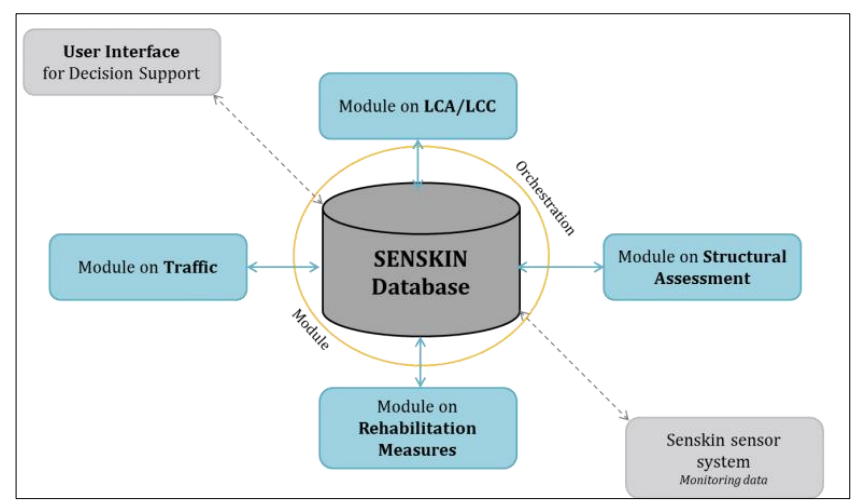

Figure 1. SENSKIN RPM - Structural layout

The Structural Assessment Module converts the measured strain of the sensors into damage states. The module on Rehabilitation Measures identifies 
rehabilitation options for each bridge element and each damage condition. Their life cycle-related environmental and economic impacts are evaluated in the LCC/LCA submodule, which constitutes the core module of the RPM. In addition, the traffic module calculates external environmental and socio-economic effects caused by the impact of remediation measures on transport. These external effects are combined with the results derived from the LCA/LCC submodule. Following, the Structural Assessment Module as well as the LCA/LCC submodule are described in more detail, outlining their scientific and functional backgrounds.

\subsection{The Structural Assessment Module}

The purpose of the Structural Module is to assess the structural condition and safety of the monitored structural members in the bridge superstructure and reinforced concrete piers, exclusively based on measurements of the SENSKIN sensors attached on the beams and piers surfaces in correspondence of the critical sections. The results of the strain measurement are processed taking into consideration the loading system at the time of installation, shrinkage and creep effects on existing materials. The cross sections of the monitored elements are then analyzed based on the constitutive laws of the materials in order to assess the actual structural capacity of the investigated bridge element under operating loads. Furthermore, a stochastic assessment is implemented in order to assess the structural reliability and the probability of failure as a function of time considering the time-dependent deterioration of the materials. The structural capacity of the bridge elements is also assessed taking into consideration future changes in the loading system due to the application of updated regulations, or the event of oversize loads travelling the bridge. Finally the SENSKIN system assesses the structural behaviour of the structural elements of the bridge under seismic loads and horizontal forces based on the sensors measurements through the displacement analysis of the structural elements. The uncertainty in structural behaviour is taken into account by modelling the load effects on the structure, the strength and toughness and the sensor measurements as random variables.

\subsection{LCA/LCC Submodule}

Within the LCA/LCC submodule, direct and external environmental effects as well as related costs for bridge rehabilitation options are determined for a time period of 50 years. Aggregated results (per $1 \mathrm{~m}^{2}$ of bridge surface area and per year) are displayed for the whole time period and detailed results are presented in 5 year steps. For comparison reasons and based on the maintenance described in DIN EN 13306 [4] and rehabilitation scenarios set up within the German project NaBrü [5], three scenarios are chosen:

- a basic scenario where rehabilitation measures are only executed when the structural element reaches a high damage state and therefore lifespans of the structural elements are assumed to be shorter;

- a preventive scenario where rehabilitation measures are conducted when the structural element reaches a low or medium damage state and therefore lifespans of the structural elements are assumed to be longer; 
- and the own scenario where the critical damage state and lifespan of each structural element can be chosen by the end-user.

Rehabilitation options have been set up for each structural element, e.g. for steel beams there are four rehabilitation options available where one of it is 'welding additional steel elements'. The default mapping of damage states and rehabilitation options was gathered alongside the structural assessment module.

Thanks to the structure of the RPM, which is kept quite open, the update of background data for future analysis (e.g. cost, environmental and traffic data) is possible very easily.

\subsubsection{LCA/LCC methodology and system boundaries}

The SENSKIN methodology for environmental life cycle based decision support within bridge rehabilitation planning is based on the method of Life Cycle Assessment (LCA) according to ISO 14040/14044 [6], [7]. LCA is a method which assesses and quantifies the associated environmental impacts of products, services or systems over their entire life cycle. Starting with the mining of resources, LCA takes into account the environmental impacts of intermediate products, the manufacturing and the use phase. Recycling and End-of-Life of the products, services or systems is considered as well. By including the whole life cycle the so called "shift of burdens" - optimizing one life cycle stage or environmental impact at the expense of increasing the environmental impacts of another life cycle stage of environmental impact - is avoided.

Main regulatory framework for life cycle costing considerations is the ISO 15686 part 5 [8] to which SENSKIN is referring to. This standard sets boundaries for life cycle costing on building level. Requirements and specifications made within this standard are adopted for bridge life cycle costing. The ISO standard provides a cost classification which differentiates clearly between Whole-life cost (WLC) and life cycle costs. Life cycle costs are defined as costs over the physical, technical, economic or functional life of the constructed asset, over a defined period of analysis. Together with non-construction costs, incomes and externalities, life cycle costs constitute the whole-life costs of the constructed asset according to the ISO standard. The constructed asset for SENSKIN is defined as the bridge rehabilitation option and is the object of assessment. The dynamic investment calculation and the net present value method are used for the LCC in the SENSKIN project.

The incorporated life cycle phases for assessment are based on the classification according to DIN EN 15978 [9], defining specifications and requirements for the life cycle-based sustainability assessment of buildings as a European standard document. This classification has been adapted for bridge assessment in former research projects [5]. As the SENSKIN research project deals with the assessment of bridge rehabilitation options and their external effects, the scope includes the life cycle modules B1 (maintenance), B2 (restoration), B3 (reinforcement) and $\mathrm{E}$ (external effects).

For environmental impact assessment during LCA, the impact category Global Warming Potential (GWP) was selected on the basis of end-user requirements and its relevance to the system under investigation. The impact category offers 
scientifically proven calculation methods and is internationally recognised. It is based on its contribution to the greenhouse effect (climate warming) and is expressed in the unit 'kg $\mathrm{CO}_{2}$ - equivalent'.

\subsubsection{Consideration of external effects}

As part of the environmental and cost analysis within SENSKIN, the internal and external effects of bridge refurbishment measures are calculated. While the internal effects result from the rehabilitation measures itself, the external effects derive from traffic restrictions, such as road closures or traffic jams, required for some of the rehabilitation activities. Both the additional driving time and the longer distance travelled have economic effects, which are translated into monetary values in terms of delays and additional fuel consumption. In addition due to the traffic restrictions, higher emissions are generated, which feed into the life cycle asessment calculations. Thus, the LCA methodology also covers both the environmental impact of bridge rehabilitation options (internal effects) and additional emissions due to traffic restrictions (external effects).

The external effects are included for the lifetime of a bridge, taking into account the traffic restrictions of the individual rehabilitation options as well as the development of the energy carrieres and the transport composition within the next 50 years for every country within the European Union [10].

\subsection{Integrated Decision Support System}

The integrated Decision Support System (DSS) invokes the structural assessment modules in chapter 0 and the LCA/LCC submodule in chapter 2.3 as required and display, via the user interface, all necessary data to the end user (the bridge operator). For this, a graphical interface is able to create a 3D representation of the monitored bridge and to display on this representation in a graphical form the strains provided by the SENSKIN monitoring system on the bridge. Additionally, the end user will be able to see the strains under different scenarios of hypothesized situations (e.g., earthquakes, higher future loads) and warnings for abnormal situations. Furthermore, the end user will be able to see when and how to intervene.

\section{Validation and benchmarking in Greece}

A systematic approach will be followed to validate and benchmark the SENSKIN monitoring system and the integrated package under actual/operational field conditions. It will cover technical feasibility, economic and operational benefits analysis, the assessment of price/performance and benchmarking.

On the basis of pilot testing at the Bosporus 1 suspension bridge in Istanbul (including also benchmarking with a conventional monitoring system), a refined version of the entire SENSKIN monitoring system will be installed at the steel composite Egnatia Bridge G4 in Greece (Figure 1). In total, 64 sensors will be patched on 7 midspan and near supports sections of the superstructure as well as on the basement of two piers. For validation purpose, conventional electrical 
foil strain gages will be installed as well. Furthermore, static and dynamic load testings will accompany the field trials.

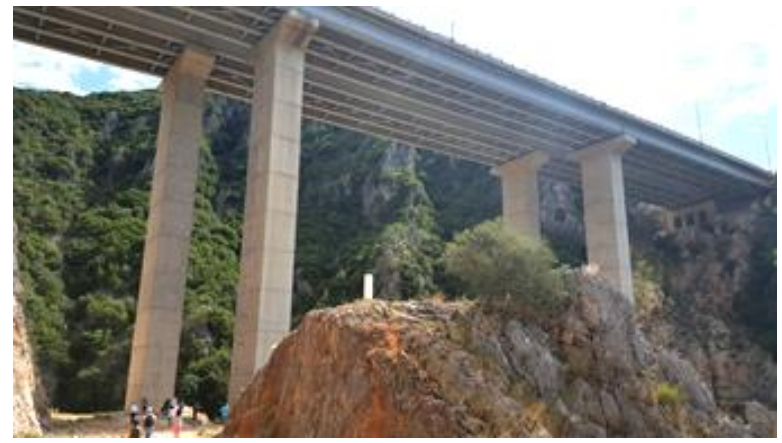

Figure 1. Egnatia Motorway G4 Bridge (@ EOAE)

From end-user and life cycle based rehabilitation planning perspective, the developed SENSKIN Rehabilitation Planning Module (RPM) will be evaluated in terms of trustworthiness, user friendliness and ease of application during the field tests in Greece. Hereby, constantly monitored sensor data will be incorporated into the SENSKIN RPM and its LCA/LCC submodule to further calibrate and benchmark the single decision support system (DSS) modules as well as the DSS itself, by using real data from Greece. Furthermore a vibration based identification of the actual mechanical performance of the bridge by using the equipment (accelerometers) and the methodology of Egnatia Odos AE [11] will test and calibrate structural assessment and DSS modules.

In that way, field testing in Greece supports the prediction of the future intervention needs, their related environmental and economic impacts [12] as well as on time detection of severe structural degradation of the bridge by including future traffic load increase.

\section{Conclusion and future Outlook}

An important outcome of the SENSKIN project, especially in terms of the growing demand for transport, is the development of an integrated monitoring system that is open to multimodal infrastructures, combined with an application-oriented DSS that supports economic and environmental analyses throughout the bridge's entire life cycle. This system will enable the reduction of maintenance costs and the associated environmental impacts for different bridge types. Hence, it is expected to contribute to the creation of a less disrupted transport infrastructure, as inspections and maintenance work can be planned more effectively. Additionally, the functioning of these infrastructures will be less affected by their increased security and resilience to growing transport demand, climate change and disruptive events. Since permanent monitoring provides precise structural data of the strains, necessary rehabilitation measures can be developed and carried out to extend the service life of bridges. Thus, the SENSKIN project helps to plan and establish effective maintenance methods for bridges. By taking into 
account sustainability aspects of bridge maintenance, the rehabilitation planning system contributes to a sustainable infrastructure and supports decision makers in the proactive life cycle based planning of bridge rehabilitation.

\section{Acknowledgements}

The research leading to these results has received funding from the EC H2020 project SENSKIN (Contract N.635844). Authors would like to thank all partners within the SENSKIN consortium.

\section{References}

[1] Salokangas, Lauri (2013): ETSI Project (Stage 3). Bridge Life Cycle Optimisation. Hg. v. Alto University, School of Engineering, Dept. of Civil and Structural Engineering. Helsinki University of Technology. Helsinki, Finnland.

[2] Du, Gangli (2015): Life cycle assessment of bridges, model development and case studies. Doctoral Thesis. Hg. v. Royal Institute of Technology (KTH). Royal Institute of Technology (KTH). Stockholm, Sweden.

[3] Safi, Mohammed Abed El-Fattah (2013): Life Cycle Costing. Applications and Implementations in bridge Investment and Management. Doctoral Thesis. Hg. v. Department of Civil and Architectural Engineering, Division of Structural Design and Bridges. Royal Institute of Technology (KTH). Stockholm, Sweden.

[4] DIN EN 13306:2010. Maintenance - Maintenance terminology. CEN/TC 319.

[5] Holistic assessment of steel and composite bridges according to criteria of sustainability (NaBrü). Project funded by AiF- German Federation of Industrial Research Associations, FOSTA/AIF P843; 2010-2012.

[6] DIN EN ISO 14040:2006. Environmental management - Life cycle assessment Principles and framework. ISO/TC 207.

[7] DIN EN ISO 14044:2006. Environmental management - Life cycle assessment Requirements and guidelines. ISO/TC 207.

[8] Standard ISO 15686-5:2017-07: ISO 15686-5 Buildings and constructed assets Service-life planning - Part 5: Life cycle costing.

[9] DIN EN 15978:2011. Sustainability of construction works - Assessment of environmental performance of buildings - Calculation method. CEN/TC 350.

[10]Capros P., De Vita A., Tasios N., Siskos P., Kannavou M., Petropoulos A., Evangelopoulou S., Zampara M., et al.: EU Reference Scenario 2016 - Energy, transport and GHG emissions Trends to 2050. European Commission Directorate General for Energy, Directorate - General for Climate Action and Directorate - General for Mobility and Transport, Luxembourg. 2016.

[11]Ntotsios E., Panetsos P. et al. (2009). Structural Identification of Egnatia Odos bridges based on ambient and earthquake induced vibrations. Bulletin of Earthquake Engineering, 7(2), pp. 485-501.

[12]P. Panetsos, S. Lambropoulos. Life -cycle maintenance cost prediction for concrete bridges based on inspection data, April 2014, TRA 2014 Conference proceedings, Paris, France. 